\title{
Plasma Source Ion Trap Mass Spectrometry: Enhanced Abundance Sensitivity by Resonant Ejection of Atomic Ions
}

\author{
G. C. Eiden, C. J. Barinaga, and D. W. Koppenaal \\ Atomic and Molecular Chemistry Section, Pacific Northwest National Laboratory, Richland, Washington, USA
}

An experimental study of resonant ion excitation in an $\mathrm{rf}$ quadrupole ion trap is reported. Atomic ions are generated in an inductively coupled plasma and injected into the ion trap where, after collisional cooling, they are irradiated by a low-voltage, dipole coupled waveform. Single frequency, narrowband, and broadband excitation pulses have been used. Absorption lineshapes (plots of observed ion signal versus excitation frequency) are shown for variations in buffer gas pressure and the amplitude and duration of the single frequency pulses. The absorption lineshapes are usually asymmetric and tail toward lower frequencies. At sufficiently low buffer gas pressure or potential well depth, the lineshapes broaden and become more asymmetric to the point that absorption by ions with adjacent mass-to-charge ratios overlaps. This overlapping absorption reduces the selectivity with which a single mass-to-charge ratio ion can be excited and ejected relative to nearby mass-to-charge ratio ions. The rate of ion ejection is different on the low versus high frequency edges of the absorption lines. This difference in ejection rates provides an important key to understanding the shape of the absorption lines. All of these observations are explained in terms of the known kinematic behavior of ions in real traps, that is, traps with substantial higher order symmetry components in the trapping field ("nonlinear" fields). The importance of the nonlinearity of the trapping field in understanding the observed lineshapes and their time dependencies is discussed. We also report resonant ejection results obtained using multiple frequency (narrow or broad bandwidth) excitation. Multiple frequency excitation allows ions with different mass-to-charge ratio values to be ejected from the trap using one excitation waveform. The finite ion storage capacity of the ion trap is thereby reserved for the ion(s) of interest. We show that ejection of ${ }^{89} \mathrm{Y}$ ions can be $\sim 10^{5}$ times more efficient than ejection of ions at either $m / z 88$ or 90 . (c) 1996 American Society for Mass Spectrometry (J Am Soc Mass Spectrom 1996, 7, 1161-1171)

$\mathrm{W}$ e have recently described the performance of a new elemental mass spectrometer-the plasma source ion trap (PSIT) [1, 2]. The PSIT is constructed from a conventional inductively coupled plasma mass spectrometer (ICP/MS) and an ion trap mass spectrometer (ITMS). Two major improvements of the PSIT compared with conventional ICP /MS were reported: elimination of most plasma-derived polyatomic ions, for example, $\mathrm{ArH}^{+}, \mathrm{Ar}_{2}^{+}, \mathrm{ArO}^{+}$, and $\mathrm{ArCl}^{+}$, and a dramatic reduction in the number of $\mathrm{Ar}^{+}$ ions observed. In addition, the contribution of ion source fluctuations, for example in laser ablation ICP/MS [3], to the fluctuations in measured isotope ratios may be reduced because all isotopes of any element can be trapped simultaneously. The advantages of the inductively coupled plasma (ICP) ion source are retained: stable and high ionization efficiency and sensitivity for nearly all elements. Almost any atomic ion can be trapped for measurements of

Address reprint requests to Dr. G. C. Eiden, Pacific Northwest National Laboratory, P.O. Box 999, M.S. P8-08, Richland, WA 99352. spectroscopic properties, reaction rate constants, or other chemical-physical properties. The PSIT is a versatile instrument for studies of isolated atomic ions. Recent improvements in the performance of the ion trap mass spectrometer [4-9] further enhance the potential of the PSIT.

Several authors have pointed out the need to discriminate against highly abundant ions when the ITMS is used to analyze laser plumes [10-12], glow discharges [13], or inductively coupled plasmas [1, 2] because the ITMS does not function well with more than $\sim 10^{4}$ trapped ions, as shown in this work and elsewhere [14]. This number is the number of ions detected at the ITMS's operational limit; the number of ions trapped is probably $2-5$ times greater. Note that this limit is $2-3$ orders of magnitude lower than the storage limit of the ITMS $\left(10^{6}-10^{7}\right.$ ions) [15]. In an ICP ion source, analyte ions are produced at trace levels compared with the major plasma ions $\mathrm{O}^{+}, \mathrm{Ar}^{+}, \mathrm{ArH}^{+}$, $\mathrm{Ar}_{2}^{+}$, and so forth. The $\mathrm{Ar}^{+}$current in PSIT is much lower than in conventional ICP/MS, but still substantial. Thus, to reserve the useful capacity of the ITMS 
for the ions of interest, high selectivity for storage of analyte ions versus $\mathrm{Ar}^{+}$ions must be realized.

We have recently reported a method to reduce or eliminate many of the major plasma ions $\left(\mathrm{Ar}^{+}, \mathrm{ArH}^{+}\right.$, $\mathrm{Ar}_{2}^{+}, \mathrm{O}^{+}$) in the PSIT and in conventional ICP/MS by reaction with $\mathrm{H}_{2}[16-18]$. However, it is not just the plasma ions that pose a problem. Even if plasma ions could be eliminated, the sample itself may contribute numerous interfering ions or the analyte of interest may be present with isotopes in very large, nonnaturally occurring ratios $\left(>10^{5}\right)$. Thus, selective ion accumulation is necessary for trace elemental analysis of most realistic samples. Our preliminary results on selective ion accumulation in the PSIT recently were reported $[19,20]$. Improvements in absolute sensitivity and abundance sensitivity were noted by using either single or multiple frequency resonant excitation waveforms to effect selective ejection of trapped atomic ions. We define the selectivity of the ejection process as the ratio of the fraction of targeted ions retained to the fraction of nontargeted ions ejected. "Abundance sensitivity" is a measure of a mass spectrometer's response to low abundance ions in the presence of high abundance ions at adjacent mass-to-charge ratios. In these experiments, ions at $m / z M$ are targeted for resonant ejection. The concomitant loss of ions at $m / z M \pm 1$, $\pm 2 \mathrm{u}$, and so forth is measured along with the loss at $m / z M$. Resonant excitation of a large ion cloud in the trap will perturb other trapped ions, especially those closest in mass-to-charge ratio to the resonantly excited ions. Realization of high selectivity in ejection of a targeted ion at $m / z M$ versus ions at adjacent massto-charge ratio $(\Delta M= \pm 1)$ represents a worst case scenario in this regard. Real samples often will have matrix ion mass-to-charge ratio values much further removed from the mass-to-charge ratio of the ions of interest, for example, analysis of actinides from a soil matrix.

Resonant excitation of ion motion in an ion trap is not a new idea [21-31]. Resonant excitation has been employed for collisionally induced dissociation (CID) in tandem mass spectrometry [21, 25, 29, 32-36], in combination with the mass selective instability scan to improve mass resolution $[6,9]$, and to extend the mass range of the ion trap [7]. Most recently, single frequency and broadband excitation have been used to selectively eject ions to enhance the accumulation and detection of the analyte(s) of interest in complex samples [37-48]. High ejection selectivity for analyte ions of interest can be realized if the absorption is sharp, that is, the widths of the absorption lineshapes are narrow compared to the spacing of the lineshape maxima for adjacent mass-to-charge ratio ions. Absorption lineshapes in this work are plots of the observed ion signal (at a given mass-to-charge ratio) versus the frequency of the resonant excitation waveform. If conditions are not optimal, the absorption can be broad enough that several mass-to-charge ratio ions are simultaneously excited, which thereby degrades selectivity. Doroshenko and Cotter [48] have reported single ion selectivity for mass-to-charge ratios up to $\sim 1300$. To understand how to obtain a sharp absorption for realistic samples so that highly selective excitation can be effected, a detailed and quantitative understanding of resonant excitation in the ion trap is of fundamental importance.

Absorption lineshapes based on calculated trajectories can be compared quantitatively with experimentally determined lineshapes only if the response of a trapped ion cloud can be measured for an arbitrary degree of excitation, from slight (no ion dissociation or ejection) to utmost (all ions ejected). Obviously, if the detection method used to characterize the excitation involves collisionally induced dissociation (CID) of molecular ions (as in most early studies [21, 25, 29, 33, $34,49-53$ ) or atomic ion ejection (as in this study), strong excitation is required. Thus, although a theoretical description of resonant excitation might include both weak and strong excitations, these experimental methods are only sensitive to strong excitation. The CID-based method to characterize resonant absorption is further limited in that the degree of dissociation depends on molecular properties as well as trapping conditions. This convolution of internal ion properties with the kinetic response of the ion cloud makes it more difficult to quantify the characteristics of resonant excitation. For elemental analysis purposes, our interest is to eject specific atomic ions from the trap or to prevent their becoming trapped. Atomic ion ejection-based absorption lineshapes do not depend on internal properties of the ion, except possibly on the polarizability [54], although this is likely a very small effect [55]. They thus provide a more direct measure of the absorption of the ion cloud than CID. Furthermore, atomic ejection is not constrained to as low an excitation fluence (the product of the amplitude and duration of the excitation pulse) or to $q$ values as low as in CID, that is, conditions that yield efficient CID without ejection, yet give stable trapping of daughter ions.

In this work, absorption lineshapes are obtained by resonant ejection of some fraction of a trapped ion cloud and plotting the remaining signal for a given mass-to-charge ratio versus resonant excitation frequency. When the excitation is on-resonance, there is a dip in the observed ion signal. Furthermore the ion cloud is cooled before resonant excitation so that it is initially near the center of the trap. Freshly injected ions show a broadened frequency response if they are not cooled before excitation [20]. If simultaneous injection and resonant excitation are used [14, 43], the resonant excitation behavior of cooled ions provides only a qualitative guide for optimization of selective ion accumulation.

Different methods to measure absorption lineshapes are potentially sensitive to the motion of the trapped ion cloud in different regions of the trapping field. In a 
pure quadrupole trap, the field strength varies linearly with displacement of the ion cloud $[15,56]$. In real traps, higher order multipoles are important--sometimes dominant-in determination of mass spectrometer performance. Thus, features of CID-based lineshapes that depend on the nonlinear field components might be expected to be more pronounced if dissociation occurs nearer to the trap's edge. Molecules with large bond dissociation energies require greater excitation fluence for dissociation; that is, strongly bound ions are expected to dissociate when they are excited far from the center of the trap where nonlinear field components are more dominant. An atomic ion ejection-based lineshape requires the full range of ion motion from the center to the edge of the trap. Such lineshapes should thus be more similar to the CIDbased lineshapes of strongly bound molecular ions than of weakly bound ions. We observe just the opposite when the lineshapes in this work were compared with some CID-based lineshapes [57].

In this article, further details of our initial resonance excitation observations $[19,20]$ are reported. We present single frequency absorption lineshapes and their dependence on the excitation fluence. Lineshapes are determined by atomic ion ejection, in contrast to most earlier work. The time dependence of the ejection process is measured by variation of the excitation duration at a fixed excitation frequency. This time dependence provides insight into the ion kinematics that is not available from the absorption lineshapes alone. The results are discussed with reference to the current understanding of resonant excitation in the rf quadrupole ion trap $[4,15,21-27]$. Preliminary multifrequency excitation results are presented; implications of the single frequency results for the design of multifrequency resonant excitation schemes are discussed.

\section{Experimental}

In the following description of the apparatus and experimental conditions, only those aspects that are significantly different from our earlier report are discussed [1,2]. The major components of this instrument are (1) an ICP ion source, (2) an ion sampling vacuum interface, (3) a linear quadrupole mass filter, (4) an ion trap mass spectrometer, and (5) a broadband waveform generator. The system is thus a standard ICP/MS with an ion trap (and einzel lens) inserted between the linear quadrupole mass filter and the channeltron detector. Ions that exit the linear quadrupole are focused onto the ion trap entrance electrode (an ITMS exit endcap electrode was used for the entrance electrode). Ions are gated into the trap by using the center element of the focusing lens and the standard gate voltage in the ITMS electronics (after inversion of the control logic). The ion trap experimental timing sequence consists of the following events with the duration of each event given in parentheses: (1) ion injection at some $q_{z}$ value (1-100 ms); (2) collisional cooling (5-30 ms); (3) resonant excitation (0.1-30 ms); (4) a radiofrequency high-voltage ramp with resonance excitation for mass analysis of the trapped ions $(0.21$ ms per unit mass-to-charge ratio scanned). The true duration of a given step in the scan function is within $0.2 \mathrm{~ms}$ of the software set-point value. The resonant excitation times given subsequently are the software set-point values.

We characterize the frequency content of the resonant excitation waveforms as either single frequency or multifrequency. The latter also can be classified with respect to the bandwidth of the waveform as either narrowband or broadband. By "narrowband," we mean those waveforms whose frequency components lie in a range on the order of the separation of the resonant frequencies of adjacent mass-to-charge ratio ions. Broadband waveforms contain a range of frequencies that correspond to the resonant frequencies of a large range of mass-to-charge ratios. The analysis rf ramp step employed an axial modulation voltage in the range $1-4 \mathrm{~V}_{\mathrm{p}-\mathrm{p}}$. Ion injection and cooling occurred at a low mass cut-off of $55 \mathrm{u}$; this value was chosen as a compromise value that gave good trapping efficiency for a wide mass range. Resonant excitation waveforms were applied to the endcap electrodes by using dipolar coupling at $q_{z}=0.2-0.6$, with much of the reported data at $q_{z} \sim 0.45$. It should be noted that $q_{z}$ values given herein are nominal values calculated from the low mass cutoff used in the scan function and with $q_{\text {eject }}=0.908$ assumed.

Three different kinds of sample solutions have been used: (1) $\mathrm{Cd}$ in $1 \% \mathrm{HNO}_{3}$ at $0.1-0.5 \mathrm{ppm}$; (2) a mixture of $1 \mathrm{ppb}$ each of $\mathrm{Sr}$ and $\mathrm{Zr}$ with $100-\mathrm{ppb} \mathrm{Y}$ in $1 \%$ $\mathrm{HNO}_{3}$; (3) a solution that contains a dozen or so elements from $\mathrm{Li}$ to $\mathrm{U}$ in concentrations between a few parts per billion and $\sim 1 \mathrm{ppm}$. These solutions are delivered to the ICP torch through an ultrasonic nebulizer at $0.5-1.0 \mathrm{~mL} / \mathrm{min}$. Mass spectra have been recorded by using the Ion Catcher Mass Spectrometer (ICMS) software [58] with the ITMS electronics or by digitizing the electrometer output with a digital storage oscilloscope (model 9310M, LeCroy, Chestnut Ridge, NY). Digitizing rates were $0.5-2.0$ Msamples $/ \mathrm{s}$, or 20-80 points across each mass peak [ $\sim 40 \mu$ s full width at half maximum (FWHM)]. Mass spectra were averaged for 50-200 scans (a scan is obtained each time the trap contents are mass selectively ejected and detected) by using the ICMS program and its key sequence programming capability.

Absorption lineshapes are plots of the observed signal at a given mass-to-charge ratio versus the frequency of the resonant excitation waveform. Plots of the observed signal versus the duration of the resonant excitation will be referred to as excitation time curves. These curves are obtained in a similar fashion to the absorption lineshapes, except that the excitation duration is varied at a fixed excitation frequency, rather 
than the converse. Excitation time curves are obtained at key frequencies across the observed lineshape. Mass spectra are recorded for discrete linear variations of excitation amplitude, duration, or frequency. To identify variables that interact with each other, absorption lineshapes were obtained at various values of $q_{2}$ (data not shown), excitation fluence, and buffer gas pressure. Step sizes were 50 or $100 \mathrm{~Hz}$ for the absorption lineshapes and $0.1-1.0 \mathrm{~ms}$ for the excitation time curves. Ten to twenty scans were averaged at each data point in the absorption lineshapes and excitation time curves. The excitation frequency was varied over a range broad enough to excite several different mass-to-charge ratio ions in turn (5-15 kHz, dependent on $q_{z}$ ) so that a single data acquisition file contains absorption lineshape data for several ions. Similarly, a single data file recorded for variation of excitation duration gives the number of targeted ions (resonantly excited) ejected as well as the number of nontargeted ions (off-resonantly excited) ejected.

Single frequency waveforms were generated by the ITMS frequency synthesizer; narrowband and broadband waveforms were generated by an HST-1000 (Teledyne Electronic Technologies, San Jose, CA). Waveforms provided with the HST-1000 are composed of preset equally spaced frequency components whose amplitudes are user selectable; further details of this method have been reported previously [37, 43]. Several different waveforms were used in this work; the frequency spacing in the various waveforms was 100 , 250,500 , or $1000 \mathrm{~Hz}$. For a given waveform, only the amplitude of each frequency component can be varied; the frequency spacing and phase relations are fixed. The phase relationships among the various frequency components are different for each waveform. Preliminary data on the effects of phase variation in narrowband excitation shows a weak, but measurable, effect on the ejection signal versus excitation duration [19].

Absorption lineshapes for narrowband excitation cannot be measured as conveniently as for single frequency excitation because computer control of the HST-1000 generated waveforms is not integrated with the ICMS program. We have attempted to measure narrowband lineshapes by variation of the amplitude of the trapping field while a narrowband excitation waveform is applied across the endcaps. These experiments have been inconclusive so far.

Helium was used as the buffer gas at a pressure of $2.0 \times 10^{-4}$ torr (indicated pressure outside the trap, 1.4 mtorr corrected; pressure inside the trap is estimated to be 2-10 times greater). Some data were acquired at pressures a factor of 2 higher or lower than this. In these experiments, effects on the absorption lineshapes were obtained for changes in the buffer gas pressure, the total charge in the trap, and the frequency, amplitude, and duration of the resonant excitation pulse. The effects of buffer gas pressure and the value of $q_{z}$ during resonant excitation have not been studied as systematically as other parameters.

\section{Results and Discussion}

One application of this work is highly selective accumulation of a given mass-to-charge ratio ion, several different mass-to-charge ratio values, or even several ranges of mass-to-charge ratios in a complex sample. All unwanted ions are ejected resonantly simultaneously by application of a waveform that contains a single frequency or a narrow range of frequencies for each ion to be ejected. However, to begin we revisit the simpler problem of single-frequency resonant excitation. These results then will guide the design of multifrequency resonant excitation schemes.

\section{Observed Lineshapes and Time Dependence for Single Frequency Excitation}

Resonant ejection has been characterized in this work by measurement of variations in the absorption lineshape for changes in buffer gas pressure, and the amplitude, duration, and spectral content of the resonant excitation pulse (single or multifrequency). The effects of some of these parameters on lineshapes are shown in Figures 1 and 2. The asymmetric lineshapes shown in Figures 1 and 2 are very similar to those observed by other workers $[27,31,57,59,60]$. Excitation of an ion of mass $M$ at the peak of its absorption also results in excitation of ions at mass $M-1$, due to the low frequency tail in the lineshapes (bold arrow,

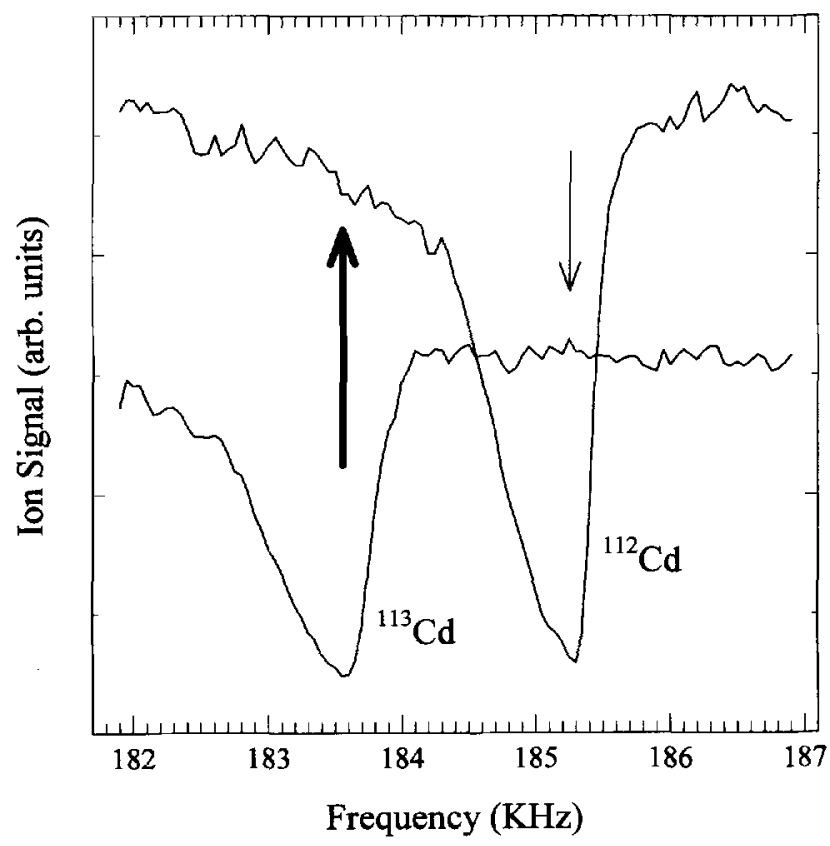

Figure 1. Resonant absorption lineshapes for ${ }^{112} \mathrm{Cd}$ and ${ }^{113} \mathrm{Cd}$ $\left(q_{z}=0.45\right.$ for $\left.{ }^{112} \mathrm{Cd}\right)$. The low frequency tail (bold arrow) in the ${ }^{112} \mathrm{Cd}$ lineshape leads to low selectivity in resonantly exciting ${ }^{113} \mathrm{Cd}$ relative to ${ }^{112} \mathrm{Cd}$. The direction of this asymmetry leads to greater excitation of ions $1 \mathrm{u}$ lower than the targeted ion compared with ions $1 \mathrm{u}$ higher. Excitation of ${ }^{112} \mathrm{Cd}$ at $\sim 185.3 \mathrm{kHz}$ (light arrow) is much more selective. Excitation amplitude and duration were $400 \mathrm{mV}_{\mathrm{p}-\mathrm{p}}$ and $5 \mathrm{~ms}$, respectively. 


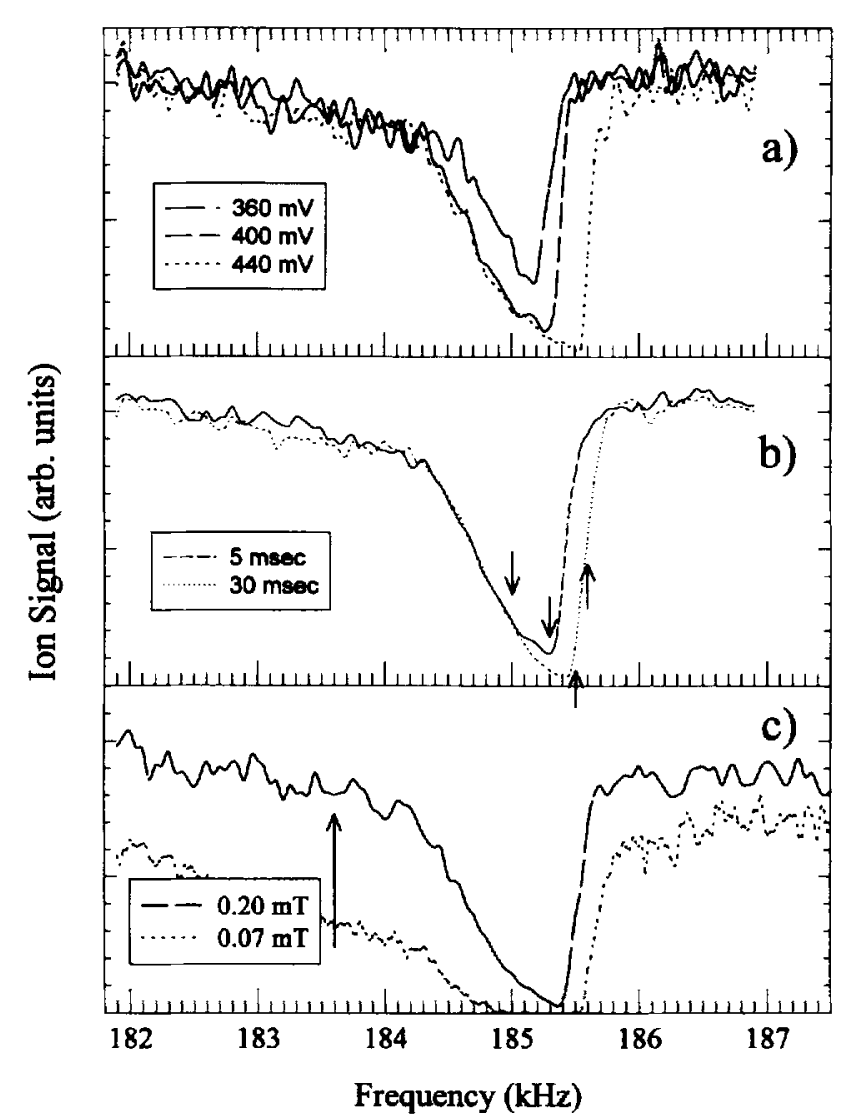

Figure 2. Atomic ion resonant ejection-based lineshapes for ${ }^{112} \mathrm{Cd}$ at $q_{z}=0.45$. (a) Excitation amplitudes are 360,400 , or 440 $\mathrm{mV}_{\mathrm{p}-\mathrm{p}}$; the curves saturate at $\sim 400 \mathrm{mV}_{\mathrm{p} \text { p }}$ (excitation duration 5 $\mathrm{ms}$ ). (b) Excitation duration 5 versus $30 \mathrm{~ms}$ at $400-\mathrm{mV}_{\mathrm{p}-\mathrm{p}}$ amplitude; the low frequency edge saturates at 2-5 ms; the arrows indicate the excitation frequencies used in Figure 3. (c) Indicated He pressure 0.07 or 0.20 mtorr; 20 -ms excitation duration at 400 $\mathrm{mV}_{\mathrm{p}-\mathrm{p}}$ (ion injection times adjusted to yield approximately constant total trapped charge). Note the poor resonant excitation selectivity at low pressure (absorption maximum of ${ }^{113} \mathrm{Cd} \sim$ $183.6 \mathrm{kHz}$; see Figure 1).

Figure 1). In contrast, excitation at mass $M-1$ results in far less excitation at mass $M$ (light arrow, Figure 1). The low frequency tails in the Figure 1 lineshapes are less pronounced at higher $q_{z}[20]$.

Figure 2 shows how the lineshapes depend on buffer gas pressure and excitation fluence. The lineshapes typically show a very gradual fall (more ions ejected) in the lowest frequency part of the tail (182.0-184.3 $\mathrm{kHz}$ in Figure 2), followed by a more sharply falling edge $(184.3-185.4 \mathrm{kHz})$, and then a rapid increase in signal at high frequency. The low frequency tails shown in Figures 1 and 2 are less pronounced at larger $q_{z}$ (data not shown) and more pronounced at low pressure (Figure 2c). The area of the low frequency tail increases dramatically for a factor of $\sim 2$ decrease in pressure. Excitation selectivity is thus poor at low pressure (the arrow in Figure $2 \mathrm{c}$ at $183.6 \mathrm{kHz}$ shows the maximum absorbance frequency for ${ }^{113} \mathrm{Cd}$ ). As expected, the number of ions ejected increases as the excitation amplitude or duration is increased (Figure
$2 a$ and $b$, respectively), but the behavior is different at low versus high frequency (Figure 3 ). Note that the low frequency edges of the curves in Figure $2 a$ and $b$ saturate with increasing fluence (at $\sim 400 \mathrm{mV}_{\mathrm{p}-\mathrm{p}}$ and $\sim 5 \mathrm{~ms}$, respectively), whereas the high frequency edge does not.

The preceding behavior is consistent with current understanding of the kinematic behavior of a resonantly excited ion cloud. The finite width of the observed lineshape is due to the inherent dispersion in the frequency response of a single ion as well as to the spatial and energy dispersion of the ion cloud. The asymmetry in the lineshape is due to the nonlinear character of the trapping field $[23,56,61-65]$. Resonant absorption in a cooled ion cloud occurs at the secular frequency of the ion cloud $\omega_{\mathrm{s}}$, where $\omega_{\mathrm{s}}$ is taken to be the frequency of maximum absorption for an ion cloud at the trap's center. If the trap has a significant octopole component in the trapping field, for example, as in a stretched trap [55, 64], the resonant frequency increases as the cloud is displaced from the center of the trap. Absorption near $\omega_{\mathrm{s}}$ causes the cloud to expand in direct proportion to the excitation duration. The rate of expansion is greatest at $\omega_{\mathrm{s}}$ and lower for frequencies higher or lower than $\omega_{s}$. If the excitation frequency is lower than $\omega_{\mathrm{s}}$, then as the cloud expands, the secular frequency increases, moving away from the applied frequency. If the excitation frequency is low enough, then the ion cloud will detune from resonance as the ion cloud expands to some critical size; that is, the ion cloud expands only to the point that absorption cuts off, as pointed out by Charles et al. [21]. Excitation at frequencies greater than $\omega_{\mathrm{s}}$ again causes expansion of the ion cloud, but at a superlinear rate. The shift in secular frequency is now toward the applied frequency and the initially weak absorption becomes stronger as the ion cloud expands. The ion cloud remains in resonance over a broader range of motion between the center and edge of the trap. If the fluence is low so that the cloud does not expand very far from the center of the trap, then the pure quadrupolar lineshape will not be distorted. If the fluence is high, then the cloud will expand toward the edges of the trap where nonlinear absorption is strong. Since high fluence is required for ion ejection, we expect ion ejection-based lineshapes to be strongly asymmetric, as observed.

Figure 3 shows the excitation duration dependence of the number of ions ejected at various excitation frequencies. Note that the vertical scale in Figure 3 is logarithmic. At low frequencies (below $184.3 \mathrm{kHz}$ in Figure 3), there is an abrupt break in slope with very little further loss of ions beyond a given excitation duration. At high excitation frequencies (Figure 3c), the signal shows a smoother variation with excitation duration. There is no slow loss period and the signal loss does not saturate for increases in excitation amplitude or duration. At yet higher excitation frequencies (Figure 3d), the rate of ion ejection is nearly the same at short and long excitation duration. The transition 


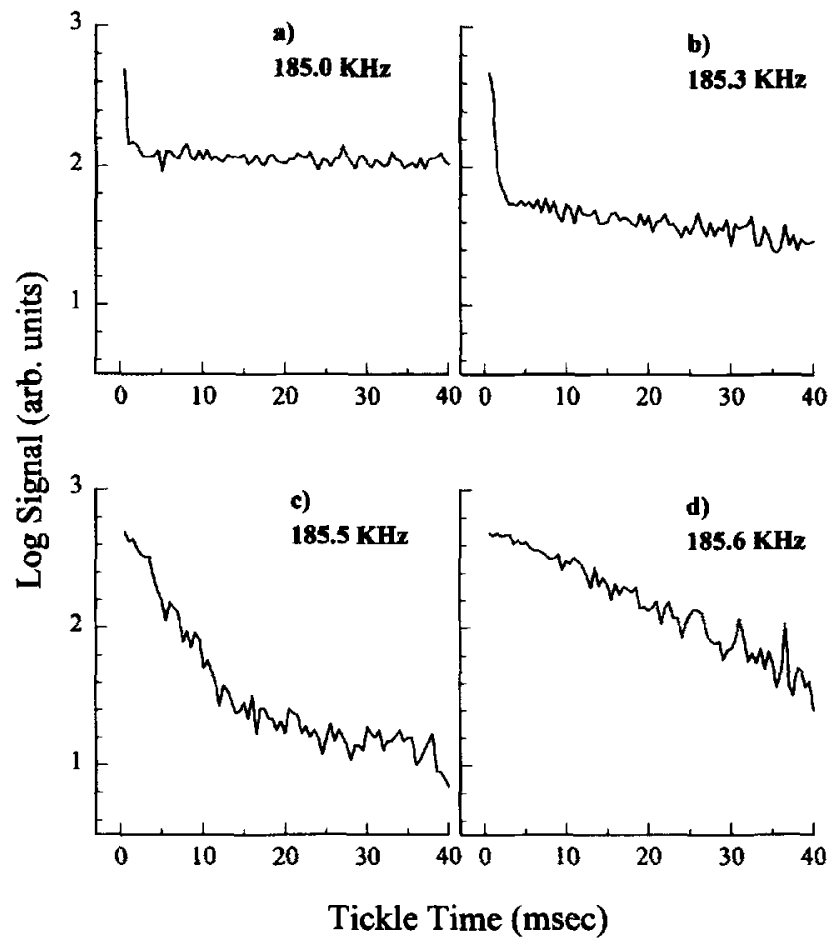

Figure 3. Semilog plots of the ${ }^{112} \mathrm{Cd}$ ion signal versus excitation duration that shows different behavior at low versus high excitation frequencies (arrows in Figure 2b). The transition from the behavior in (a) to that in (d) occurs over a frequency range of $\sim 300 \mathrm{~Hz}$. At frequencies below the transition frequency $(\sim 185.4$ $\mathbf{k H z}$ ), the ejection rate beyond a few milliseconds is nearly zero. Above the transition frequency, the ejection rate at long excitation duration is substantial. Greater overall ejection efficiency is obtained at high frequency and long excitation duration. Excitation amplitude is $400 \mathrm{mV}_{\mathrm{p} \text {-p }}$; buffer gas pressure $0.2 \mathrm{mtorr}$.

from low to high frequency behavior in the shapes of the curves in Figure 3 occurs over a frequency interval (185.3-185.5 kHz in Figure 3) that is narrow compared to the width of the lineshapes; this interval coincides with the absorption maxima in Figures 1, 2, and 4. Behavior similar to that shown in Figure 3 also has been observed for ${ }^{112} \mathrm{Cd}$ resonantly excited at a $q_{z}$ value of 0.59 .

As reported by Franklin et al. [66] and others [21, $26,33]$, the observed time dependence can be divided into three periods: an induction period during which little or no ion loss is observed; a period of rapid ion loss; a period of slow ion loss. Franklin et al. [66] associated the induction period with the minimum fluence required to eject ions from the trap. The induction period is not observed in the low time resolution data (1-ms time step) in Figure 3; however, an induction period of $\sim 0.5 \mathrm{~ms}$ was observed in similar data recorded at higher time resolution (not shown). In the rapid loss portion of the curves in Figure $3 a-c$, the minimum fluence for ejection has been reached and a significant loss of ions occurs. The magnitude of this loss depends on the excitation amplitude. The slow ion loss period is more difficult to explain (see subsequent text).
Several mechanisms have been invoked by previous workers to explain the leveling off of the ejection rate versus excitation duration. Charles et al. [21] ascribed the leveling off to a balance of power absorption from the rf field with power dissipation via collisions. Power absorption is expected to decrease as ions are excited away from the center of the trap due to the shift of the resonant frequency in nonlinear trapping fields, as noted before. Also, as the ion cloud is excited out to the boundaries of the trapping field, that is, as the fluence approaches the threshold for ejection, ions with the highest kinetic energy and/or largest orbits will be lost first. This initial loss of ions has several effects. Lower space-charge results in an increase of the resonant frequency of the remaining ions; this effect is expected to be on the order of $1 \mathrm{~Hz}$ for a loss of $\sim 50$ ions [31], or a maximum of $\sim 20 \mathrm{~Hz}$ for the largest number of ions used in the present study. With fewer ions trapped, the coulombic repulsive force on each ion is decreased, so that greater fluence should be required to eject the last ions that leave the trap compared to the first ones out. Considering the similarity of the absorption lineshape measured for an approximately fivefold increase in space-charge [20], this effect appears to be small. Space-charge effects reinforce the effect of the field nonlinearity to increase the resonant frequency of ions displaced from the center of the trap, namely, ejection of the last ions out of the trap is suppressed. However it does not appear that these factors can explain the orders-of-magnitude change in slope shown in Figure 3a.

The different time dependence observed for the low frequency versus high frequency edges of the lineshape may be due in part to the increase in secular frequency as the cloud is displaced from the center of the trap. For discussion purposes, we take the secular frequency to be $\omega$ for the excited cloud and $\omega_{\mathrm{s}}$ for the cooled cloud at the center of the trap (pure quadrupole value); $\omega>\omega_{\mathrm{s}}$ for a symmetrically stretched trap [55, $64,67]$. The observed absorption lineshape can be considered to be a sum of a pure quadrupole absorption band (absorption at $\omega_{\mathrm{s}}$ near the center of the trap) and a nonlinear absorption band (absorption at $\omega>\omega_{\mathrm{s}}$ near the edges of the trap). The rationale for use of a broad low intensity lineshape for the quadrupole absorption and a narrow high intensity lineshape for the nonlinear absorption is as follows. First, the quadrupole resonance has been calculated to lie at a lower frequency than nonlinear resonances [55, 64, 67]. Second, excitation at frequencies above the secular frequency of the cooled ion cloud (i.e., ions at the center of the trap) leads to a superlinear growth in the displacement of the ion cloud [23]. Such nonlinear phenomena observed in diverse fields of study show more sharply peaked frequency response than linear phenomena, for example, single versus multiple photon absorption. Hence, there is an expectation that the nonlinear resonance will be narrower (FWHM) than the linear resonance. These features correspond to the observed ab- 
sorption lineshapes, that is, we observe a broad low intensity feature on the low frequency edge of the absorption lines.

Figure 4 shows a fit of the ${ }^{112} \mathrm{Cd}$ lineshape to a sum of two gaussians. The individual component bands from the fit are shown by the solid lines, their sum by the dashed line, and the experimental data by the symbols. Ejection at $\omega_{\mathrm{s}}$ is rapid because the cloud expands linearly in time in the quadrupolar band. For excitation at $\omega$, the initial displacement of the ion cloud is due to weak absorption in the high frequency tail of the quadrupolar band and the displacement increases more slowly than for excitation at $\omega_{\mathrm{s}}$. As the cloud is displaced from the center of the trap, absorption becomes stronger and the rate of displacement eventually surpasses the rate at $\omega_{\mathrm{s}}$.

We suggest that the abrupt change in slope in the excitation duration curves (Figure 3 ) at low excitation frequency is due to two factors: the dependence of $\omega$ on displacement and the phase space distribution of the ion cloud. Resonant excitation near the ejection threshold implies that, at a given fluence, some ions are above threshold while others are below the threshold. For excitation at low frequency, the absorbance of the ion cloud decreases with increasing displacement. Thus, only the most weakly trapped ions are ejected, that is, those with the largest orbits and/or highest kinetic energies. For the remaining ions, the fluence is below threshold because they lie deeper in the trapping well [15]. The effect of the resonant ejection pulse is to depopulate the most weakly trapped portion of

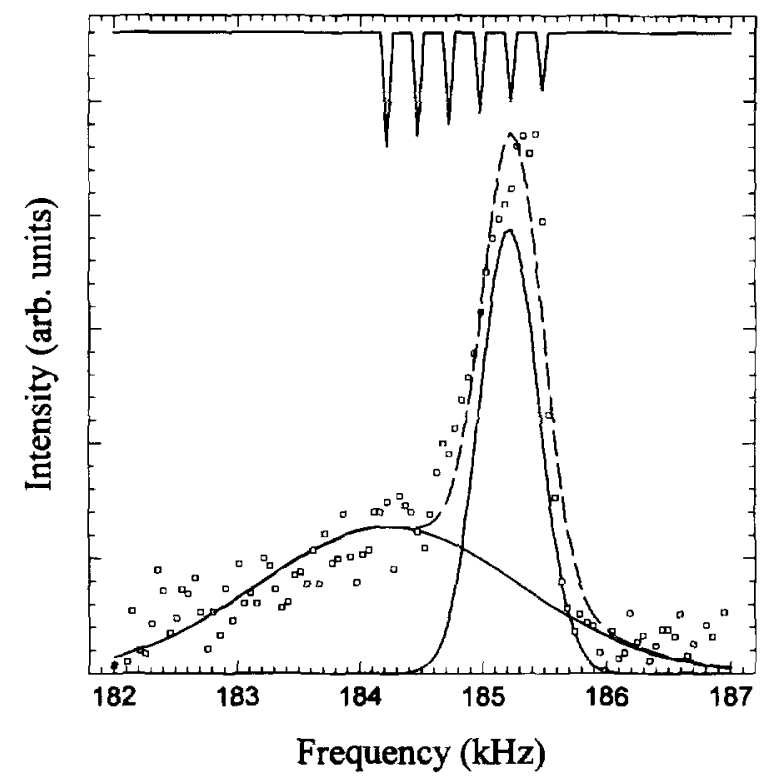

Figure 4. Proposed model of resonant absorption lineshapes. An experimental atomic ion ejection-based lineshape is shown with a fit to overlapping absorption bands: a weak low frequency band due to the purely quadrupolar trapping field a strong high frequency band due to the nonlinear portion of the trapping field. The model suggests a form (inverted peaks in the top trace) for the amplitude envelope for high selectivity in narrowband excitation of a single mass-to-charge ratio. the ion phase space distribution. Apparently, the return to the steady state distribution is slow on the time scale of the current experiments, that is, $>30 \mathrm{~ms}$ in Figures $3 a-d$ (note that the slope in Figure 3a, after the break, is not quite zero). This relaxation time is approximately an order of magnitude longer than the collisional cooling time measured by Hemberger et al. [68]. The observed difference in the time dependence at low versus high excitation frequency is therefore suggested to be due to a combination of the ease of ejection of the most weakly trapped ions and the displacement dependence of the secular frequency of the cloud.

By using these ideas, we can now suggest a form for the envelope of amplitudes of the frequency components used to excite each mass-to-charge ratio ion in multifrequency resonance ejection (or CID). The amplitudes of the low frequency components in a frequency window that corresponds to a single mass-to-charge ratio should be larger than the higher frequency components. This is shown schematically in the topmost trace in Figure 4. This scheme places the bulk of the resonant excitation waveform intensity farthest from the absorption maximum of adjacent mass-to-charge ratio ions. This situation also may lead to the most rapid ejection of ions, which is consistent with ultrahigh selectivity in ion ejection. This behavior has been explored in preliminary narrowband excitation experiments on the $\mathrm{Ag}-\mathrm{Cd}$ system $[19,69]$, but no systematic analysis yet has been made. Cooling of the ion cloud should preferentially reduce the absorption intensity in the low frequency wing of the line and thereby enhance the selectivity of the excitation.

This work also has shown that resonant excitation with unit mass resolution or better requires moderately high buffer gas pressures. The trade-offs inherent in optimization of the buffer gas pressure with respect to the trapping efficiency of injected ions and resonance ejection selectivity could be alleviated either by controlling the buffer gas pressure on a short time scale by using a pulsed valve or by not relying on buffer gas collisions to effect ion trapping. The latter can be accomplished by using phase synchronous injection, which has been modeled extensively [70, 71], or dynamic rf trapping, which has been demonstrated experimentally [12]. However, these methods are most appropriate for pulsed ion sources, where the pulse duration is short compared to the period of the $\mathrm{rf}$ trapping field, or to the risetime of the amplitude of the $\mathrm{rf}$ trapping potential, respectively. We are pursuing experiments to measure the phase dependence of trapping in the PSIT and methods to efficiently couple a continuous ion source into the ITMS.

\section{Abundance Sensitivity and Multiple Frequency Excitation}

Preliminary studies of trapped and cooled metal ions have shown that ions of mass $M$ can be ejected with 
$\sim 10^{5}$ times the efficiency of ions at mass $M \pm 1[19$, 20]. In this work, resonant excitation during ion injection has not been characterized as fully as for trapped and cooled ions; the selectivity is significantly lower in the former case. To achieve the highest possible selectivity, some of the issues raised by earlier reports on resonant excitation are revisited.

In Figure 5, the selectivity with which a single mass-to-charge ratio ion can be ejected by narrowband resonant excitation is shown. Figure 5 a shows a mass
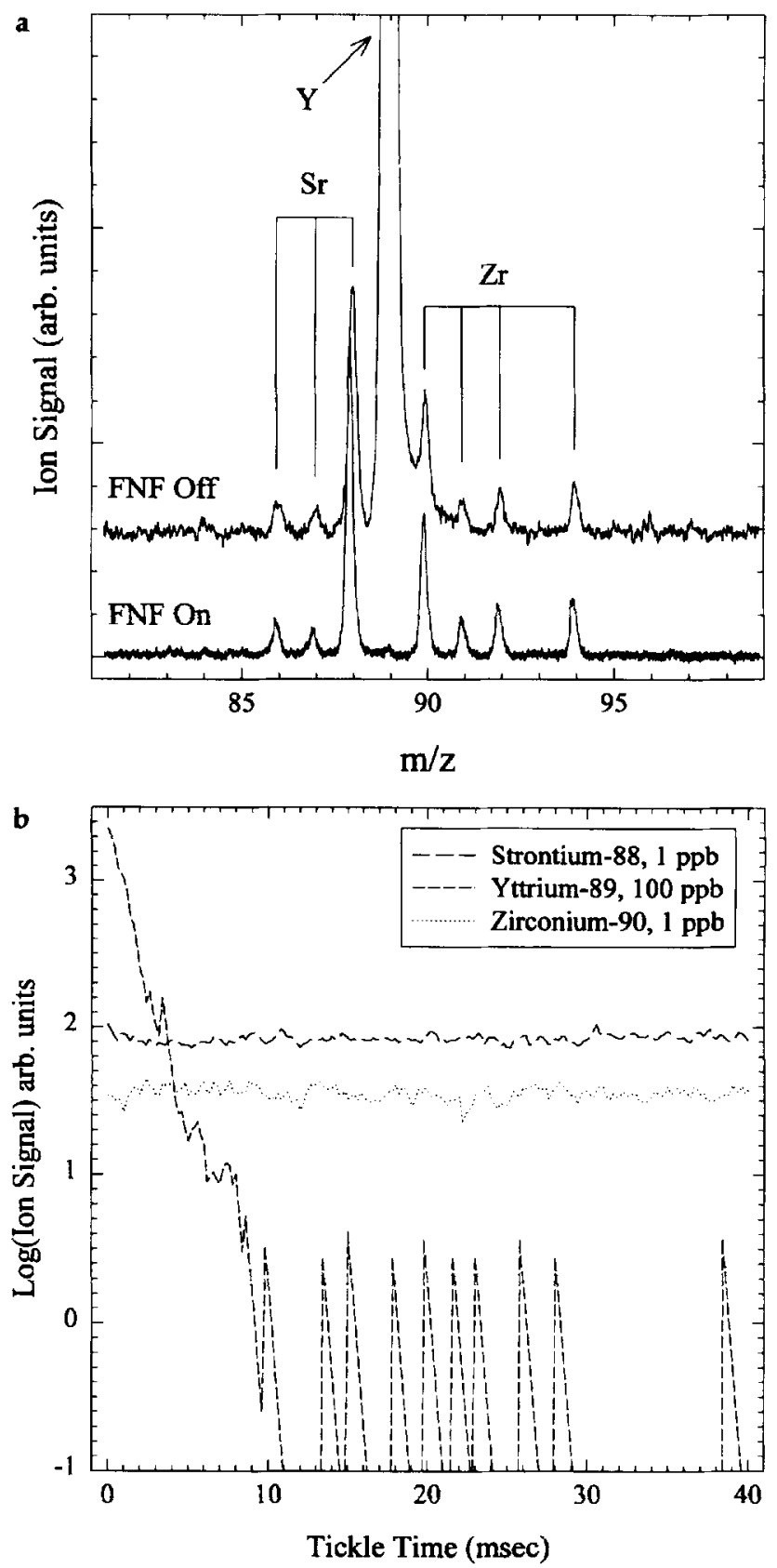

Figure 5. (a) $\mathrm{Sr}-\mathrm{Y}-\mathrm{Zr}$ mass spectrum with narrowband resonant excitation on (bottom trace) and off (top trace). The narrowband waveform contains three frequency components (10-ms duration). (b) A semilog plot of the ${ }^{88} \mathrm{Sr},{ }^{89} \mathrm{Y}$, and ${ }^{90} \mathrm{Zr}$ signals in (a) versus excitation duration that shows the $\sim 10^{5}$ selectivity possible by using resonant ejection. spectrum of $\mathrm{Sr}, \mathrm{Y}$, and $\mathrm{Zr}$ obtained with (bottom trace) and without (top trace) the resonant ejection waveform applied. The waveform was composed of three frequency components $(255.50,255.75$, and $256.00 \mathrm{kHz})$ of equal amplitude. The ${ }^{88} \mathrm{Sr},{ }^{90} \mathrm{Zr}$, and ${ }^{89} \mathrm{Y}$ concentrations were $0.8,0.5$, and $100 \mathrm{ppb}$, respectively. The $Y$ signal is reduced approximately two thousandfold between the upper and lower traces; the difference in signal-to-noise ratio in the two traces is due to different digitizer gain settings used to obtain these data. The $\mathrm{Sr}$ and $\mathrm{Zr}$ signals in the bottom trace show the same intensity as in spectra of a solution that contains only $\mathrm{Sr}$ and $\mathrm{Zr}$ (without resonant excitation); that is, selective ejection of $Y$ before the mass analysis scan restores the $\mathrm{Sr}$ and $\mathrm{Zr}$ peaks to the intensities they would have had if no $Y$ were in the sample. Figure $5 b$ shows the signal versus excitation duration data for resonant excitation of $\mathrm{Y}^{+}$; the $\mathrm{Y}^{+}$signal is reduced by a factor of $\sim 2000$ in only $10 \mathrm{~ms}$ (fraction retained $\sim 1 / 2000$ ). The ${ }^{88} \mathrm{Sr}$ and ${ }^{90} \mathrm{Zr}$ signals are unaffected to within experimental error. The upper limit for the $\mathrm{Sr}$ and $\mathrm{Zr}$ loss is taken to be a few percent based on the error in the slope of a best fit line to the data in Figure $5 b$ (fraction ejected $<1 / 50$ ). This gives a selectivity of $\sim 10^{5}$ for ejecting the target ion $\left(\mathrm{Y}^{+}\right)$compared with the nontargeted ions $\left(\mathrm{Sr}^{+}\right.$, $\left.\mathrm{Zr}^{+}\right)$in adjacent mass channels. Corresponding improvements in abundance sensitivity may be possible.

Broadband resonant ejection of large background ion populations enhances the sensitivity of the ion trap mass spectrometer [37, 42-45]. Most of the demonstrations of this enhancement have focused on examples from organic and biological mass spectrometry, where interfering ions often occur over a large $(\sim 1000-u)$ mass range. The mass spectrum for a large number of ions spread over such a large mass range shows ion peaks broadened and distorted by the high space-charge conditions in the trap. Selective ion accumulation [ejection of all trapped ions except the ion(s) of interest] results in a lower background and higher mass resolution, that is, much higher signal-to-noise ratio. The reported signal-to-noise enhancement is between 1 and 3 orders of magnitude, dependent on the sample and experimental conditions. The selectivity of resonant ejection decreases as the difference in mass between the ions of interest and the interfering ions decreases. An extreme is reached in the measurement of large isotope ratios, where the minor isotope must be measured in the presence of the space-charge of the major isotope. Figure 6 shows the PSIT performance when a mixture that contains a large range of elements (analyte masses of 7-238 in the sample, although substantial signal was not observed below $m / z \sim 60$ ) is used; this provides a better comparison to the literature reports of enhanced sensitivity for complex organic samples.

Without broadband excitation, approximately $10^{4}$ ions are detected. The data in Figure 6 show the severity of the distortion of the mass spectrum under these space-charge conditions. Without selective ejec- 


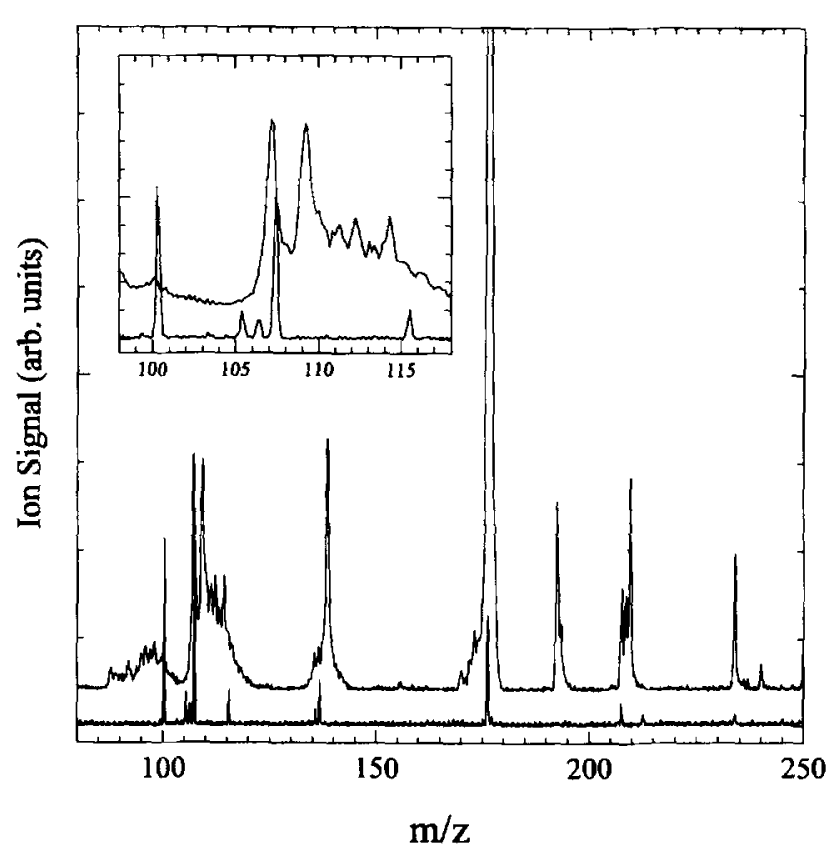

Figure 6. Space-charge effects in the ITMS (multielement solution; $m / z \quad 7-238$; concentrations between 10 and $1000 \mathrm{ppb}$ ). Without broadband excitation (top trace), $\sim 10^{4}$ ions are detected. Minor isotopes are barely discernible; $m / z 105$ and 106 are completely obscured (inset). With broadband excitation (bottom trace), space-charge is reduced and $m / z \quad 100-106$ ions are detected at the same count rates as in an undistorted mass spectrum recorded under low space charge conditions.

tion, the ${ }^{100}$ Mo ion peak is barely discernible and $\mathrm{m} / \mathrm{z}$ 105 (a hydrocarbon peak) and ${ }^{106} \mathrm{Cd}$ are completely obscured (inset). With selective ejection tailored to retain only ions of $m / z 100-107$, full sensitivity is recovered for $m / z$ 100-106 (lower trace). This observation was confirmed by measuring the spectrum under lower space-charge conditions where good peak shapes were obtained for $m / z$ 100-107. Sensitivity for ${ }^{107} \mathrm{Ag}$ in the lower trace in Figure 6 is not as good as under lower space-charge conditions, possibly due to incomplete optimization of the position of the frequency notch. The broadband waveform amplitude used was the minimum necessary to recover a nonspace-charged mass spectrum in the region of interest; this resulted in detection of some ions outside the $\mathrm{m} / \mathrm{z}$ 100-107 window as shown in the lower trace.

\section{Conclusions}

This work extends previous studies of dipolar resonant absorption of ions trapped in the ITMS and has demonstrated that the frequency and time dependencies of atomic ion ejection provide a powerful means to gain greater insight into the mechanism of resonant excitation. The data presented are consistent with earlier reports except for the variation of the excitation duration curves with excitation frequency. Experimentally observed lineshapes and their time dependence have been rationalized by using the known kinematic behavior of ions in nonlinear traps. A model has been suggested that describes the asymmetric lineshapes as a sum of two absorption bands, which are due to the linear and nonlinear components of the trapping field. In this model, different excitation duration dependencies observed for low versus high excitation frequency have been ascribed to the spatial and energy distribution of the ion cloud and to the position dependence of the resonant frequency in a nonlinear trapping field. The major features of the atomic ion ejection-based lineshapes observed experimentally thus can be accounted for by using recent descriptions of ion motion in nonlinear traps.

Selective ion ejection for the ion trap mass spectrometer also has been characterized by using narrowband and broadband resonant excitation. The abundance sensitivity of the ion trap mass spectrometer is improved by several orders of magnitude by using broadband excitation as compared with a total ion accumulation mode. The single frequency absorption results provide a qualitative guide to optimize the amplitudes of the frequency components in multifrequency resonance excitation. The lineshape model suggested in this work provides a rationale for designing multifrequency excitation waveforms, namely, in the excitation bandwidth that corresponds to a single mass-to-charge ratio, the amplitude of the low frequency components should be larger than those of the higher frequency components. This places the bulk of the excitation intensity farthest from the low frequency tail of lower mass-to-charge ratio ions. This also may lead to the most rapid ejection of ions consistent with ultrahigh selectivity in ion ejection by prompt excitation of ions into the strongly nonlinear portion of the trapping field. This behavior has been explored in preliminary narrowband excitation experiments on the Ag-Cd system [69], but no systematic analysis has been made yet.

\section{Acknowledgments}

The authors thank Dr. Nathan Yates and Dr. Donald Eades for several helpful discussions of this work and other unpublished results. We are grateful also to the reviewers for many helpful suggestions. This research was conducted at Pacific Northwest National Laboratory which is operated for the U.S. Department of Energy by Battelle under Contract DE-AC06-76RLO 1830. The authors acknowledge financial support for this work from the Department of Energy (Office of Research and Development and Laboratory Technology Applications Group) and the Finnigan Corporation through a Cooperative Research and Development Agreement. GCE also would like to acknowledge funding for a Postdoctoral Research Fellowship from Associated Western Universities, Inc.-Northwest Division under Grant DE-FG06-89ER75522 from the U.S. Department of Energy.

\section{References}

1. Barinaga, C. J.; Koppenaal, D. W. Rapid Commun. Mass Spectrom. 1994, 8, 71 . 
2. Koppenaal, D. W.; Barinaga, C. J.; Smith, M. R. I. Anal. At. Spectrom. 1994, 9, 1053.

3. Allen, A. A.; Pang, H. M.; Warren, A. R.; Houk, R. S. J. Anal. At. Spectrom. 1995, 10, 267.

4. March, R. E.; Todd, J. F. J., Eds. Practical Aspects of Ion Trap Mass Spectrometry; CRC Press: Boca Raton, FL, 1995; Vols. 1-3.

5. Londry, F. A.; March, R. E. Int. J. Mass Spectrom. Ion Processes $1995,144,87$

6. Schwartz, J. C.; Syka, J. E. P.; Jardine, I. J. J. Am. Soc. Mass Spectrom. 1991, 2, 198.

7. Kaiser, R. E.; Cooks, R. G.; Stafford, G. C.; Syka, J. E. P.; Hemberger, P. H. Int. J. Mass Spectrom. Ion Processes 1991, $106,79$.

8. Williams, J. D.; Cox, K. A.; Cooks, R. G.; Kaiser, R. E.; Schwartz, I. C. Rapid Commun. Mass Spectrom. 1991, 5, 327.

9. Londry, F. A.; Wells, G. J.; March, R. E. Rapid Commun. Mass Spectrom. 1993, 7, 43.

10. Gill, C. G.; Blades, M. W. J. Anal. At. Spectrom. 1993, 8, 261.

11. Garrett, A. W.; Hemberger, P. H.; Nogar, N. S. Spectrochim. Acta, Part B, 1995, 50, 1889.

12. Eiden, G. C.; Garrett, A. W.; Cisper, M. E.; Nogar, N. S.; Hemberger, P. H. Int. J. Mass Spectrom. Ion Processes 1994, 136,119 .

13. Duckworth, D. C.; Barshick, C. M.; Smith, D. H.; McLuckey, S. A. Anal. Chem. 1994, 66, 92.

14. Duckworth, D. C.; Smith, D. H.; Goeringer, D. E.; McLuckey, S. A. Proceedings of the 43rd ASMS Conference on Mass Spectrometry and Allied Topics; Atlanta, GA, 1995; p 41.

15. March, R. E.; Hughes, R. J. Quadrupole Storage Mass Spectrometry; Wiley: New York, 1989.

16. Eiden, G. C.; Barinaga, C. J.; Koppenaal, D. W. Proceedings of the 1996 Winter Conference on Plasma Spectrochemistry. ICP Information Newsletter 1996, 21, 179.

17. Eiden, G. C.; Barinaga, C. J.; Koppenaal, D. W. J. Anal. At. Spectrom. 1996, 11, 317.

18. Koppenaal, D. W.; Barinaga, C. J.; Eiden, G. C. Proceedings of the 1996 Winter Conference on Plasma Spectrochemistry. ICP Information Newsletter 1996, 21, 261.

19. Eiden, G. C.; Barinaga, C. J.; Koppenaal, D. W. Proceedings of the 43rd ASMS Conference on Mass Spectrometry and Allied Topics; Atlanta, GA, 1995; p 1104.

20. Eiden, G. C.; Barinaga, C. J.; Koppenaal, D. W. Proceedings of the 43rd ASMS Conference on Mass Spectrometry and Allied Topics; Atlanta, GA, 1995; p 983.

21. Charles, M. J.; McLuckey, S. A.; Glish, G. L. J. Am. Soc. Mass Spectrom. 1994, 5, 1031.

22. Alfred, R. L.; Londry, F. A.; March, R. E. Int. J. Mass Spectrom. Ion Processes 1993, 125, 171.

23. Franzen, J. Int. J. Mass Spectrom. Ion Processes 1994, 130, 15.

24. March, R. E.; Londry, F. A.; Alfred, R. L; Todd, J. F. J.; Penman, A. D.; Vedel, F.; Vedel, M. Int. J. Mass Spectrom. Ion Processes 1991, 110, 159.

25. Paradisi, C.; Traldi, P.; Vettori, U. Rapid Commun. Mass Spectrom. 1993, 7, 440.

26. March, R. E.; Weir, M. R.; Tkaczyk, M.; Londry, F. A.; Alfred, R. L.; Franklin, A. M.; Todd, J. F. J. Org. Mass Spectrom. 1993, 28, 499.

27. Sugiyama, K.; Yoda, J. Appl. Phys. B 1990, 51, 146.

28. Fulford, J. E.; Hoa, D.-N.; Hughes, R. J.; March, R. E.; Bonner, R. F.; Wong, G. J. J. Vac. Sci. Technol. 1980, 17, 829.

29. Louris, J. N.; Cooks, R. G.; Syka, J. E. P.; Kelley, P. E.; Stafford, G. C. J.; Todd, J. F. J. Anal. Chem. 1987, 59, 1677.
30. March, R. E.; McMahon, A. W.; Londry, F. A.; Alfred, R. L.; Todd, J. F. J.; Vedel, F. Int. J. Mass Spectrom. Ion Processes $1989,95,119$.

31. Vedel, F.; Vedel, M.; March, R. E. Int. l. Mass Spectrom. Ion Processes 1990, 99, 125.

32. Todd, J. F. J.; Penman, A. D.; Thorner, D. A.; Smith, R. D. Rapid Commun. Mass Spectrom. 1990, 4, 108.

33. Johnson, J. V.; Yost, R. A.; Kelley, P. E.; Bradford, D. C. Anal. Chem. 1990, 62, 2162.

34. Hart, K. J.; McLuckey, S. A. J. Am. Soc. Mass Spectrom. 1994, $5,250$.

35. Liere, P.; Blasco, T.; March, R. E.; Tabet, J.-C. Rapid Commun. Mass Spectrom. 1994, 8, 953.

36. McLuckey, S. A.; Goeringer, D. E.; Glish, G. L. Anal. Chem. 1992, 64, 1455.

37. Kenny, D. V.; Callahan, P. J.; Gordon, S. M.; Stiller, S. W. Rapid Commun. Mass Spectrom. 1993, 7, 1086.

38. McLuckey, S. A.; Glish, G. L.; Duckworth, D. C.; Marcus, R. K. Anal. Chem. 1992, 64, 1606.

39. McLuckey, S. A.; Goeringer, D. E.; Glish, G. L. I. Am. Soc. Mass Spectrom. 1991, 2, 11.

40. Shaffer, B. A.; Karnicky, J.; Buttrill, S. E. Proceedings of the 41 st ASMS Conference on Mass Spectrometry and Allied Topics; San Francisco, CA, 1993; p 802.

41. Soni, M. H.; Cooks, R. G. Anal. Chem. 1994, 66, 2488.

42. Garrett, A. W.; Cisper, M. E.; Nogar, N. S.; Hemberger, P. H. Rapid Commun. Mass Spectrom: 1994, 8, 174.

43. Goeringer, D. E.; Asano, K. G.; McLuckey, S. A.; Hoekman, D.; Stiller, S. W. Anal. Chem. 1994, 66, 313.

44. Guan, S. H.; Marshall, A. G. Anal. Chem. 1993, 65, 1288

45. Julian, R. K.; Cooks, R. G. Anal. Chem. 1993, 65, 1827.

46. Schubert, M.; Nagel, M.; Wang, Y.; Franzen, Y. Proceedings of the 43rd ASMS Conference on Mass Spectrometry and Allied Topics; Atlanta, GA, 1995; p 1107.

47. Karr, D.; Hoekman, D.; Koski, A. Proceedings of the 43rd ASMS Conference on Mass Spectrometry and Allied Topics; Atlanta, GA, 1995; p 1079.

48. Doroshenko, V. M.; Cotter, R. J. Proceedings of the 43rd ASMS Conference on Mass Spectrometry and Allied Topics; Atlanta, GA, 1995; p 1102.

49. March, R. E. Int. J. Mass Spectrom. Ion Processes 1992, 118, 71.

50. March, R. E.; Weir, M. R.; Londry, F. A.; Catinella, S.; Traldi, P.; Stone, J. A.; Jacobs, W. B. Can. J. Chem. 1994, 72, 966.

51. Paradisi, C.; Traldi, P.; Vettori, U. Rapid Commun. Mass Spectrom. 1993, 7, 690.

52. Plomley, J. B.; Koester, C. J.; March, R. E. Anal. Chem. 1994, 66,4437 .

53. Vettori, U.; Traldi, P. Rapid Commun. Mass Spectrom. 1993, 7, 312.

54. Bortolini, O.; Traldi, P. Rapid Commun. Mass Spectrom. 1994, 8,666 .

55. Londry, F. A.; Morrison, R. J. S.; March, R. E. Proceedings of the 43rd ASMS Conference on Mass Spectrometry and Allied Topics; Atlanta, GA, 1995; p 1124.

56. Wang, Y.; Franzen, J. J. Mass Spectrom. Ion Processes 1992, 112, 167 .

57. Williams, J. D.; Cox, K. A.; Cooks, R. G.; McLuckey, S. A.; Hart, K. J.; Goeringer, D. E. Anal. Chem. 1994, 66, 725.

58. Yost, R. A.; Yates, N. A. ICMS Ion Trap Software 2.20, Gainesville, FL, 1992.

59. Vedel, F.; Vedel, M.; March, R. E. Int. I. Mass Spectrom. Ion Processes 1991, 108, R11.

60. Vedel, F. Int. I. Mass Spectrom. Ion Processes 1991, 106, 33.

61. Franzen, J. Int. J. Mass Spectrom. Ion Processes 1993, 125, 165.

62. Wang, Y.; Franzen, J.; Wanczek, K. P. Int. J. Mass Spectrom. Ion Processes 1993, 124, 125. 
63. Wang, Y.; Franzen, J. Int. J. Mass Spectrom. Ion Processes 1994, 132, 155.

64. Franzen, J. Int. J. Mass Spectrom. Ion Processes 1991, 106, 63.

65. Wang, Y. Rapid Commun. Mass Spectrom. 1993, 7, 920.

66. Franklin, A. M.; Todd, J. F. J.; March, R. E. Proceedings of the 41st ASMS Conference on Mass Spectrometry and Allied Topics; San Francisco, CA, 1993; p 925.

67. Major, F. G.; Dehmelt, H. G. Phys. Rev. 1968, 170, 91.
68. Hemberger, P. H.; Nogar, N. S.; Williams, J. D.; Cooks, R. G.; Syka, J. E. P. Chem. Phys. Lett. 1992, 191, 405.

69. Koppenaal, D. W.; Alexander, M. L.; Barinaga, C. J.; Smith, M. R.; Eiden, G. C.; Mendoza, A. 1994 FACSS; St. Louis, MO, 1994; p 167.

70. O, C. S.; Schuessler, H. A. Int. I. Mass Spectrom. Ion Phys. 1981, 40, 53.

71. O, C. S.; Schuessler, H. A. Int. J. Mass Spectrom. Ion Phys. $1981,40,67$. 